\title{
Arrow's Theorem with a Fixed Feasible Alternative*
}

\author{
Allan Gibbard ${ }^{1}$, Aanund Hylland ${ }^{2}$, and John A. Weymark ${ }^{3}$ \\ Department of Philosophy, University of Michigan, Ann Arbor, MI 48109, USA \\ 2 Department of Economics, University of Oslo, P.O. Box 1095, Blindern, N-0317 Oslo, Norway \\ 3 Department of Economics, University of British Columbia, Vancouver, B.C., Canada V6T 1 Y2
}

Received June 4, 1986/Accepted in revised form January 15, 1987

\begin{abstract}
Arrow's Theorem, in its social choice function formulation, assumes that all nonempty finite subsets of the universal set of alternatives is potentially a feasible set. We demonstrate that the axioms in Arrow's Theorem, with weak Pareto strengthened to strong Pareto, are consistent if it is assumed that there is a prespecified alternative which is in every feasible set. We further show that if the collection of feasible sets consists of all subsets of alternatives containing a prespecified list of alternatives and if there are at least three additional alternatives not on this list, replacing nondictatorship by anonymity results in an impossibility theorem.
\end{abstract}

\section{Introduction}

Arrow's [2] Impossibility Theorem is usually expressed in terms of social welfare functions; a social welfare function assigns a social preference ordering to each admissible profile of individual orderings of a set of alternatives. The theorem, though, can also be expressed in terms of social choice functions. A social choice function does the following: for each set of alternatives that might turn out to be feasible, and for each admissible preference profile, a social choice function specifies a nonempty subset of the feasible set - the choice set. The use of a social choice function thus requires a specification of which subsets of the universal set of alternatives are potential feasible sets. In the standard choice-theoretic versions of Arrow's Theorem, the collection of feasible sets consists of all nonempty finite subsets (or at least all two and three element subsets) of the set of alternatives. Given

\footnotetext{
* Most of the research for this article was completed while we were participants in the Public Choice Institute held at Dalhousie University during the summer of 1984. We wish to record here our thanks to the Institute Director, E.F. McClennen, and its sponsors, the Council for Philosophical Studies, the U.S. National Science Foundation, and the Social Science and Humanities Research Council of Canada. We are grateful to our referees for their comments and the Center for Mathematical Studies in Economics and Management Science at Northwestern University, where Weymark was a visitor during 1985-86, for secretarial assistance.
} 
this domain assumption, Arrow's Theorem states that no social choice function with an unrestricted preference domain satisfies all of (i) the Arrow [1] choice axiom, (ii) independence of (preferences involving) infeasible alternatives, (iii) weak Pareto, and (iv) nondictatorship.

The usual proofs of Arrow's Theorem rely heavily on the assumption that any pair of alternatives is a possible feasible set, i.e. any two alternatives might turn out to be the only feasible alternatives. Bailey [3] questions the relevence of Arrow's domain assumption, and thus questions the relevance of Arrow's Theorem, for problems involving the design of economic institutions. In an economic problem, feasible sets might be known in advance, say, to take the following form: a feasible set consists of all of the allocations attainable given a particular specification of the state of technical knowledge and the initial endowment of resources. Such feasible sets typically contain an infinite number of alternatives and, thus, do not satisfy Arrow's domain assumption mentioned above. In a similar vein, Richelson [14] argues that in many political decision-making contexts, not all finite subsets of the universal set are potentially feasible. In particular, he notes that in many situations there is a status quo alternative which is in every feasible set.

It may be worthwhile, then, to consider the sensitivity of Arrow's Theorem to alternative specifications of the collection of feasible sets. Previous work on this topic has been rather limited. Grether and Plott [6] consider what they call $k$-set feasibility. With $k$-set feasibility, the collection of feasible sets consists of all finite subsets of alternatives containing at least $k$ alternatives. They demonstrate that Arrow's axioms are inconsistent with this collection of feasible sets if $k$ is strictly less than the total number of alternatives. ${ }^{1}$ Donaldson and Weymark [5], motivated by economic problems, restrict attention to feasible sets which are compact subsets of a Euclidean space and contain an infinite number of alternatives. By placing natural economic restrictions on preferences and on feasible sets of alternatives, they demonstrate that Arrow's other axioms are consistent with these domain assumptions.

In the present article we explore the implications for social choice theory of having every potential feasible set contain a prespecified list of alternatives. This restriction on the collection of feasible sets is satisfied, for example, if there is a status quo alternative which is always available. ${ }^{2}$ Similarly, this restriction is satisfied if there is a fixed alternative, not necessarily the status quo, which is adopted if no agreement is reached on the choice of another alternative, as in many bargaining situations. In many institutional settings, the rules of order require that one alternative - the motion - and (often implicitly) the status quo are always under consideration. In this example, every potential feasible set contains two fixed alternatives. ${ }^{3}$

We demonstrate that the axioms in Arrow's Theorem, with weak Pareto strengthened to strong Pareto, are consistent if the assumption that the feasible set domain is unrestricted is replaced by the assumption that there is a prespecified list

\footnotetext{
1. See Grether and Plott [6] for a discussion of earlier related contributions. Panda [12,13] extends a number of social choice theorems from an unrestricted feasible set domain to $k$-set feasibility.

2 Richelson [14] provides a number of illustrations of this phenomenon.

3 We are indebted to Bernie Grofman for suggesting this example.
} 
of alternatives which are always feasible. We do not have a complete characterization of the class of social choice functions which satisfy Arrow's axioms with this domain restriction. However, we are able to demonstrate that any member of this class of functions must exhibit some dictatorial features if the collection of feasible sets consists of all subsets of alternatives containing the prespecified list of alternatives and if there are at least three additional alternatives not on this list. Furthermore, with this strengthened domain restriction, we find that replacing nondictatorship by anonymity results in an impossibility theorem.

\section{Notation, Definitions, and Background Results}

There is a finite set of individuals $M=\{1, \ldots, m\}$ where $m \geq 2$ and a universal set of alternatives $X$ containing a finite number $n \geq 3$ of elements. Each individual has a (weak) ordering, i.e. a reflexive, complete, and transitive binary relation, $R_{i} \in \mathscr{R}$ of $X$ where $\mathscr{R}$ is the set of all orderings of $X$. For each weak preference relation $R \in \mathscr{R}$, strict preference $P$ and indifference $I$ are defined in the usual fashion. A preference profile $\left\langle R_{i}\right\rangle:=\left(R_{1}, \ldots, R_{m}\right) \in \mathscr{R}^{m}$ is a vector of individual preference orderings. The collection of admissible preference profiles is denoted by $\mathscr{D}$ and is called the preference domain. The collection of potential feasible sets is denoted by $\mathscr{S}$ and is called the feasible set domain; a member of $\mathscr{S}$ is called a feasible set.

A social choice function $C: \mathscr{S} \times \mathscr{D} \rightarrow \mathscr{P}(X)$, where $\mathscr{P}(X)$ is the set of all subsets of $X$, is a mapping which assigns to each admissible feasible set and preference profile a nonempty subset of the feasible set called the choice set, i.e. for all $S \in \mathscr{S}$ and all $\left\langle R_{i}\right\rangle \in \mathscr{D}, C\left(S,\left\langle R_{i}\right\rangle\right) \subset S$ with $C\left(S,\left\langle R_{i}\right\rangle\right) \neq \emptyset$. A social welfare function $F: \mathscr{D} \rightarrow R$ is a mapping which assigns a social preference ordering of the alternatives to each admissible preference profile. A social welfare function $F$ rationalizes a social choice function $C$ if for all $S \in \mathscr{S}$ and all $\left\langle R_{i}\right\rangle \in \mathscr{D}$,

$$
C\left(S,\left\langle R_{i}\right\rangle\right)=\left\{x \in S \mid x F\left(\left\langle R_{i}\right\rangle\right) y \text { for all } y \in S\right\} .
$$

In other words, the choice set $C\left(S,\left\langle R_{i}\right\rangle\right)$ is the set of most preferred elements in $S$ according to the social preference ordering $F\left(\left\langle R_{i}\right\rangle\right)$.

We consider feasible set domains which have a special property: every feasible set contains a fixed set $Q$ of alternatives.

$Q$-Restricted Feasible Set Domain. For $Q \subset X$ with $Q \neq \emptyset, S \in \mathscr{P} \rightarrow Q \subset S$.

Suppose, for example, as in many contexts, there is a status quo alternative $\bar{x}$ which is always feasible, then this condition is satisfied with $Q=\{\bar{x}\}$. When $Q=\{\bar{x}\}$, we write $\bar{x}$-restricted instead of $\{\bar{x}\}$-restricted. For $\emptyset \neq Q \subset Q^{\prime} \subset X$, if $\mathscr{S}$ is a $Q^{\prime}$-restricted feasible set domain, then $\mathscr{S}$ is also a $Q$-restricted feasible set domain. Thus to say that every feasible set contains the set of alternatives $Q$ does not rule out the possibility that every feasible set contains some superset $Q^{\prime}$ of $Q$.

A $Q$-restricted feasible set domain need not include every subset of $X$ containing $Q$, so it is possible for distinct collections of feasible sets $\mathscr{S}$ and $\mathscr{S}^{\prime}$ both to be $Q$-restricted feasible set domains. The collection of all subsets of $X$ containing $Q$ is called the complete $Q$-restricted feasible set domain. 
Complete $Q$-Restricted Feasible Set Domain. For $Q \subset X$ with $Q \neq \emptyset, S \in \mathscr{S} \leftrightarrow Q \subset S$.

The complete $Q$-restricted feasible set domain is not a $Q^{\prime}$-restricted feasible set domain for any $Q^{\prime} \subset X$ such that $Q \subset Q^{\prime}$ and $Q \neq Q^{\prime}$. Richelson [14] works with a complete $\bar{X}$-restricted feasible set domain.

We shall have occasion to consider a preference domain which consists of all profiles satisfying two properties: ( $i$ ) everyone prefers the alternatives not in $Q$ to the alternatives in $Q$ and (ii) for all admissible profiles, each person has a single ranking of the alternatives in $Q$, where $Q$ is a fixed set of alternatives. We denote the restriction of a preference profile $\left\langle R_{i}\right\rangle \in \mathscr{R}^{m}$ to $Q \subset X$ by $\left\langle R_{i}\right\rangle_{Q}$.

$Q$-Minimal Preference Domain. For $Q \subset X$ with $Q \neq \emptyset$ and for $\left\langle\bar{R}_{i}\right\rangle \in \mathscr{R}^{m}$, $\mathscr{D}=\left\{\left\langle R_{i}\right\rangle \in \mathscr{R}^{m} \mid\right.$ (i) for all $i \in M, x P_{i} y$ for all $x \in X \backslash Q$ and for all $y \in Q$ and (ii) $\left.\left\langle R_{i}\right\rangle_{Q}=\left\langle\bar{R}_{i}\right\rangle_{Q}\right\}$.

When $Q=\{\bar{x}\}$, we write $\bar{x}$-minimal instead of $\{\bar{x}\}$-minimal. There is only one $\bar{x}$-minimal preference domain as the second defining property of a $Q$-minimal preference domain is trivially satisfied if $Q=\{\bar{x}\}$. If $\# Q \geq 2$, there are many $Q$-minimal preference domains; each differs in the way individuals rank the alternatives in $Q$.

The remainder of the conditions we consider in our theorems are standard in the literature.

Unrestricted Feasible Set Domain. $\mathscr{S}=\mathscr{P}(X) \backslash \emptyset$.

Unrestricted Preference Domain. $\mathscr{D}=\mathscr{R}^{m}$.

Arrow's Choice Axiom. For all $S, S^{\prime} \in \mathscr{S}$, for all $\left\langle R_{i}\right\rangle \in \mathscr{D}$, if $S \subset S^{\prime}$ and if $C\left(S^{\prime},\left\langle R_{i}\right\rangle\right) \cap S \neq \emptyset$, then $C\left(S,\left\langle R_{i}\right\rangle\right)=C\left(S^{\prime},\left\langle R_{i}\right\rangle\right) \cap S^{4}$

Independence of Infeasible Alternatives. For all $S \in \mathscr{P}$, for all $\left\langle R_{i}\right\rangle,\left\langle R_{i}^{\prime}\right\rangle \in \mathscr{D}$, if $x R_{i} y \leftrightarrow x R_{i}^{\prime} y$ for all $i \in M$ and for all $x, y \in S$, then $C\left(S,\left\langle R_{i}\right\rangle\right)=C\left(S,\left\langle R_{i}^{\prime}\right\rangle\right)$.

Weak Pareto. For all $S \in \mathscr{S}$, for all $\left\langle R_{i}\right\rangle \in \mathscr{D}$, if $x, y \in S$ and if $x P_{i} y$ for all $i \in M$, then $y \notin C\left(S,\left\langle R_{i}\right\rangle\right)$.

Strong Pareto. For all $S \in \mathscr{P}$, for all $\left\langle R_{i}\right\rangle \in \mathscr{D}$, if $x, y \in S$ and if $x R_{i} y$ for all $i \in M$ with $x P_{i} y$ for some $i \in M$, then $y \notin C\left(S,\left\langle R_{i}\right\rangle\right)$.

Nondictatorship. There does not exist a $j \in M$ such that for all $S \in \mathscr{S}$, for all $\left\langle R_{i}\right\rangle \in \mathscr{D}$, $C\left(S,\left\langle R_{i}\right\rangle\right) \subset\left\{x \in S \mid x R_{j} y\right.$ for all $\left.y \in S\right\}$.

Anonymity. For all $S \in \mathscr{P}$, for all $\left\langle R_{i}\right\rangle,\left\langle R_{i}^{\prime}\right\rangle \in \mathscr{D}$, if $\left\langle R_{i}^{\prime}\right\rangle$ is a permutation of the orderings in $\left\langle R_{i}\right\rangle$, then $C\left(S,\left\langle R_{i}\right\rangle\right)=C\left(S,\left\langle R_{i}^{\prime}\right\rangle\right)$.

\footnotetext{
4 On an unrestricted feasible set domain, Arrow's choice axiom is equivalent to the weak axiom of revealed preference, which is defined as : for all $x, y \in X$, for all $\left\langle R_{i}\right\rangle \in \mathscr{D}$, if there exists an $S \in \mathscr{I}$ such that $x \in C\left(S,\left\langle R_{i}\right\rangle\right)$ and $y \in S \backslash C\left(S,\left\langle R_{i}\right\rangle\right)$, then there does not exist an $S^{\prime} \in \mathscr{P}$ such that $y \in C\left(S^{\prime},\left\langle R_{i}\right\rangle\right)$ and $x \in S^{\prime}$. See Arrow [1, Theorem 1] or Suzumura [17, Theorem 2.3]. In general, Arrow's choice axiom is weaker than the weak axiom of revealed preference. See Suzumura [17, Theorem 2.1].
} 
In terms of social choice functions, Arrow's [2] Theorem may be stated as follows. ${ }^{5}$

Arrow's Theorem. If a social choice function has an unrestricted feasible set domain and an unrestricted preference domain, then it can not satisfy all of (i) Arrow's choice axiom, (ii) independence of infeasible alternatives, (iii) weak Pareto, and (iv) non-dictatorship.

If the feasible set domain $\mathscr{S}$ has the property that for all $S, S^{\prime} \in \mathscr{S}, S \cup S^{\prime} \in \mathscr{S}$, then $\mathscr{P}$ is closed under (finite) unions. An unrestricted feasible set domain has this property as does any complete $Q$-restricted feasible set domain. The role that Arrow's choice axiom plays in Arrow's Theorem may be seen from the following proposition due to Hansson [7]. ${ }^{6}$

Hansson's Theorem. If the feasible set domain $\mathscr{S}$ is closed under finite unions, then Arrow's choice axiom is a necessary and sufficient condition for a social choice function to be rationalizable by a social welfare function.

For an unrestricted feasible set domain, the social welfare function which rationalizes a social choice function that satisfies Arrow's choice axiom is unique, and is determined from the choices made out of the two-element feasible sets. For a complete $Q$-restricted feasible set domain, not all two-element subsets of $X$ can be feasible and, in general, more than one social welfare function may rationalize a given social choice function which satisfies Arrow's choice axiom.

\section{Results}

Our possibility theorem states that the axioms in Arrow's Theorem, with weak Pareto strengthened to strong Pareto, are consistent if the assumption that the feasible set domain is unrestricted is replaced by the assumption that the feasible set domain is $\bar{x}$-restricted. ${ }^{7}$

Theorem 1. There exist social choice functions defined on an $\bar{x}$-restricted feasible set domain containing a feasible set $S$ with $\# S \geq 2$ and on an unrestricted preference domain which satisfy all of (i) Arrow's choice axiom, (ii) independence of infeasible alternatives, (iii) strong Pareto, and (iv) nondictatorship. ${ }^{8}$

\footnotetext{
5 Arrow's Theorem is also valid if the feasible set domain contains all two and three element subsets of $X$. Similarly, our Theorems 2 and 3 below are valid if all sets of alternatives containing $Q$ with cardinality $\# Q+2$ and $\# Q+3$ are feasible sets.

6 Our statement of Hansson's Theorem differs in inessential ways from Hansson's original version. The main difference arises from the fact that the choice functions Hansson considers do not include preference profiles as arguments. See also Suzumura [17, Theorem A(8)].

${ }_{7}$ Note that establishing this theorem for an $\bar{x}$-restricted feasible set domain also establishes the theorem for any $Q$-restricted feasible set domain.

8 If $\mathscr{S}=\{\bar{x}\}$, everyone is a dictator.
} 
Proof. The proof is by construction. Assume $\mathscr{S}$ is an $\bar{x}$-restricted feasible set domain. For all $x \in X$ and for all $\left\langle R_{i}\right\rangle \in \mathscr{D}$, define

$$
\begin{aligned}
& N^{+}\left(x,\left\langle R_{i}\right\rangle\right):=\#\left\{i \in M \mid x P_{i} \bar{x}\right\}, \\
& N^{-}\left(x,\left\langle R_{i}\right\rangle\right):=\#\left\{i \in M \mid \bar{x} P_{i} x\right\},
\end{aligned}
$$

and

$$
N\left(x,\left\langle R_{i}\right\rangle\right):=N^{+}\left(x,\left\langle R_{i}\right\rangle\right)-N^{-}\left(x,\left\langle R_{i}\right\rangle\right) .
$$

For all $S \in \mathscr{P}$ and for all $\left\langle R_{i}\right\rangle \in \mathscr{D}$, define

$$
\begin{aligned}
\tilde{C}^{R}\left(S,\left\langle R_{i}\right\rangle\right): & =\left\{x \in S \mid N\left(x,\left\langle R_{i}\right\rangle\right) \geq N\left(y,\left\langle R_{i}\right\rangle\right) \text { for all } y \in S\right\}, \\
\tilde{C}^{0}\left(S,\left\langle R_{i}\right\rangle\right): & =\left\{x \in \widetilde{C}^{R}\left(S,\left\langle R_{i}\right\rangle\right) \mid N^{-}\left(x,\left\langle R_{i}\right\rangle\right) \leq N^{-}\left(y,\left\langle R_{i}\right\rangle\right)\right. \\
& \text { for all } \left.y \in \tilde{C}^{R}\left(S,\left\langle R_{i}\right\rangle\right)\right\},
\end{aligned}
$$

and

$$
\begin{aligned}
\tilde{C}^{i}\left(S,\left\langle R_{i}\right\rangle\right):= & \left\{x \in \tilde{C}^{i-1}\left(S,\left\langle R_{i}\right\rangle\right) \mid x R_{i} y \text { for all } y \in \widetilde{C}^{i-1}\left(S,\left\langle R_{i}\right\rangle\right)\right\}, \\
& i=1, \ldots, m
\end{aligned}
$$

The social choice function is $\widetilde{C}^{m}$.

The satisfaction of Arrow's choice axiom is established by showing that $\widetilde{C}^{m}$ is rationalized by a social welfare function. For a given preference profile $\left\langle R_{i}\right\rangle$, the alternatives in $X$ are first ordered by the numbers $N\left(x,\left\langle R_{i}\right\rangle\right)$. Second, tied alternatives are ranked by the number of negative votes received in a pairwise comparison with $\tilde{x}$. Alternatives which are tied at this stage are then ranked by a serial dictatorial ordering. The resulting social preference relationship is a lexicographic ordering which rationalizes $\widetilde{C}^{m}\left(\cdot,\left\langle R_{i}\right\rangle\right)$. Thus, Arrow's choice axiom is satisfied by $\widetilde{C}^{m}$.

The information used to determine $\widetilde{C}^{m}\left(S,\left\langle R_{i}\right\rangle\right)$ consists solely of the individual rankings of alternatives in $S$. Hence, if the preference profile changes in such a way that the individual rankings in $S$ remain unchanged, the choice set does not change either. Consequently, independence of infeasible alternatives is satisfied by $\widetilde{C}^{m}$.

Strong Pareto is established using a proof by contradiction. Suppose $x, y \in S \in \mathscr{S}$ with $x R_{i} y$ for all $i$ and $x P_{i} y$ for some $i$ but $y \in \widetilde{C}^{m}\left(S,\left\langle R_{i}\right\rangle\right)$. Since individual preference relations are orderings, for this configuration of preferences, we must have $N\left(x,\left\langle R_{i}\right\rangle\right)=N\left(y,\left\langle R_{i}\right\rangle\right)$ and $N^{-}\left(x,\left\langle R_{i}\right\rangle\right)=N^{-}\left(y,\left\langle R_{i}\right\rangle\right)$, so $x \in \widetilde{C}^{0}\left(S,\left\langle R_{i}\right\rangle\right)$, otherwise $y$ would not be in $\widetilde{C}^{0}\left(S,\left\langle R_{i}\right\rangle\right)$. But having $\{x, y\} \subset \widetilde{C}^{0}\left(S,\left\langle R_{i}\right\rangle\right)$ is inconsistent with having an $i \in M$ such that both $x P_{i} y$ and $y \in \widetilde{C}^{i}\left(S,\left\langle R_{i}\right\rangle\right)$. Thus $\widetilde{C}^{m}$ satisfies strong Pareto.

Nondictatorship is also established using a proof by contradiction. Suppose person $j$ is a dictator. By assumption there exists an $S \in \mathscr{S}$ with at least two elements, $\bar{x}$ and $y$. By the unrestricted preference domain assumption, we can consider a profile $\left\langle R_{i}\right\rangle$ with the property that (i) $y P_{j} \bar{x}$ and $\bar{x} P_{j} z$ for all $z \in X \backslash\{\bar{x}, y\}$ and (ii) $\bar{x} P_{i} y$ and $y P_{i} z$ for all $z \in X \backslash\{\bar{x}, y\}$ for all $i \neq j$. For all $z \neq \bar{x}, y, N\left(z,\left\langle R_{i}\right\rangle\right)$ is negative. For $y, N\left(y,\left\langle R_{i}\right\rangle\right)=2-m$. Regardless of the profile, $N\left(\bar{x},\left\langle R_{i}\right\rangle\right)=N^{-}\left(\bar{x},\left\langle R_{i}\right\rangle\right)=0$. Thus, if $m>2, N\left(\bar{x},\left\langle R_{i}\right\rangle\right)>N\left(y,\left\langle R_{i}\right\rangle\right)$ for all $y \neq \bar{x}$. If $m=2, N^{-}\left(y,\left\langle R_{i}\right\rangle\right)=1$. 
Hence, regardless of the value of $m(\geq 2), \tilde{C}^{m}\left(S,\left\langle R_{i}\right\rangle\right)=\{\bar{x}\}$, which contradicts the assumption that $j$ is a dictator. As $j$ is arbitrary, this argument establishes that $\widetilde{C}^{m}$ is nondictatorial.

The social choice function $\tilde{C}^{m}$ used to prove Theorem 1 can be thought of as operating in a series of steps. First, the social choice function $\widetilde{C}^{R}$ is constructed. If not all choice sets are singletons, a tie-breaking rule is introduced yielding the social choice function $\widetilde{C}^{0}$. New tie-breaking rules are then applied sequentially until the process is terminated with $\tilde{C}^{m}$.

The social choice function $\tilde{C}^{R}$ was introduced by Richelson [14]. $N^{+}\left(x,\left\langle R_{i}\right\rangle\right)$ is the number of people who strictly prefer $x$ to $\bar{x}$ in the profile $\left\langle R_{i}\right\rangle$ and, similarly, $N^{-}\left(x,\left\langle R_{i}\right\rangle\right)$ is the number of people who strictly prefer $\bar{x}$ to $x$. If, for a given profile $\left\langle R_{i}\right\rangle$, each individual assigns one point to each alternative preferred to $\bar{x}$, zero points to alternatives indifferent to $\bar{x}$, and negative one point to alternatives judged to be worse than $\bar{x}$, then $N\left(x,\left\langle R_{i}\right\rangle\right)$ is the total number of points received by $x$. For a given preference profile $\left\langle R_{i}\right\rangle$ and feasible set $S$, Richelson's social choice function $\widetilde{C}^{R}$ chooses the alternatives which receive the highest number of points in $S$. The first tie-breaking rule retains only those alternatives among the ones chosen in the first stage which make the fewest number of people worse off compared to $\bar{x}$. Finally, a serial dictatorship is used to further narrow the remaining alternatives.

While $\tilde{C}^{R}$ by itself satisfies many desirable properties, Richelson demonstrates that $\tilde{C}^{R}$ does not satisfy weak Pareto if there exists an $S \in \mathscr{S}$ with $\# S \geq 3 .{ }^{9}$ For example, if everyone prefers $z$ to $y$ to $\bar{x}$, then both $y$ and $z$ would be chosen out of $\{\bar{x}, y, z\}$, but $z$ Pareto dominates $y$. It is for this reason that the serial dictatorship is introduced as a tie-breaking rule. A simple dictatorship would be sufficient if we merely required our social choice function to satisfy weak Pareto; the serial dictatorship is adopted to ensure that our function satisfies strong Pareto.

The second step in our example is not essential when $m>2$ or if there exists an $S \in \mathscr{S}$ with $\# S \geq 3$. If this step is omitted, person one is a dictator if $m=2$ and $\# S \leq 2$ for all $S \in \mathscr{S}$. More generally, this tie-breaking rule limits the scope of the nonanonymous part of our social choice function.

Because of the use of a serial dictatorship as a tie-breaking rule, the social choice function $\tilde{C}^{m}$ is obviously unsatisfactory. Given the domain conditions in Theorem 1 , other social choice functions exist which satisfy the Arrow axioms, but they too have dictatorial features. ${ }^{10}$ For example, such a social choice function can be constructed by first, for any feasible set and admissible profile, restricting attention to $\bar{x}$ together with the set of feasible alternatives which Pareto dominate $\bar{x}$ and by then applying a serial dictatorship to these alternatives to yield the choice set. In this example, each person has the power to veto any alternative which he or she judges to be worse than $\bar{x}$, but the tie-breaking rule is again dictatorial. If the feasible set domain $\mathscr{S}$ is $\{\bar{x}, \bar{y}\}$ - restricted, the preference domain $\mathscr{D}$ can be partitioned into $m$ subsets with the property that if two profiles coincide on $\{\bar{x}, \bar{y}\}$, then they are both in the same

\footnotetext{
9 Richelson also considers a modified version of $\tilde{C}^{R}$ which satisfies weak Pareto. It is easy to verify that this modified social choice function violates Arrow's choice axiom.

10 Formal details of the following examples are included in an earlier draft of this article which appeared as Discussion Paper No. 85-15, Department of Economics, University of British Columbia.
} 
element of the partition. For each element of the partition, a different serial dictatorship is used to determine the choice sets. By assigning each person to be the first "dictator" for one of the elements of the partition, a social choice function constructed according to this procedure spreads the power to make collective decisions throughout society. Clearly, such rules are not anonymous.

All of our examples exhibit some dictatorial features. Theorem 2 demonstrates that this state of affairs is inevitable with a complete $Q$-restricted feasible set domain and a $Q$-minimal preference domain (or any larger preference domain) provided there are at least three alternatives in $X$ not in $Q$.

Theorem 2. If a social choice function has a complete $Q$-restricted feasible set domain and a $Q$-minimal preference domain and if $\# Q+3 \leq \# X$, then it can not satisfy all of (i) Arrow's choice axiom, (ii) independence of infeasible alternatives, (iii) weak Pareto, and (iv) nondictatorship.

Proof. On the contrary, assume such a social choice function exists. By assumption $T:=X \backslash Q$ contains at least three distinct alternatives. Let $\left\langle R_{i}\right\rangle_{T}, \mathscr{D}_{T}, \mathscr{R}_{T}^{m}, S_{T}$, and $\mathscr{S}_{T}$ denote the restrictions of $\left\langle R_{i}\right\rangle, \mathscr{D}, \mathscr{R}^{m}, S$, and $\mathscr{P}$, respectively, to the set $T$. Since $\mathscr{D}$ is a $Q$-minimal preference domain, $\mathscr{D}_{T}=\mathscr{R}_{T}^{m}$, i.e. $\mathscr{D}_{T}$ is an unrestricted preference domain on $T$. Since $\mathscr{S}$ is a complete $Q$-restricted feasible set domain, $\mathscr{S}_{T}$ is an unrestricted feasible set domain on $T$. Define the social choice function $C^{*}: \mathscr{S}_{T} \times \mathscr{R}_{T}^{m} \rightarrow \mathscr{P}(T)$ by setting $C^{*}\left(S_{T},\left\langle R_{i}\right\rangle_{T}\right):=C\left(S,\left\langle R_{i}\right\rangle\right)$ for all $S \in \mathscr{S}$ and for all $\left\langle R_{i}\right\rangle \in \mathscr{D}$. Since $C$ satisfies weak Pareto and $\mathscr{D}$ is a $Q$-minimal preference domain, $C^{*}$ is a well-defined social choice function on $T$ with an unrestricted feasible set domain and an unrestricted preference domain. It is easy to confirm that $C^{*}$ satisfies (i) Arrow's choice axiom, (ii) independence of infeasible alternatives, (iii) weak Pareto, and $(i v)$ nondictatorship. But satisfaction of these conditions by $C^{*}$ violates Arrow's Theorem, a contradiction.

Suppose $X$ contains at least four alternatives and a social choice function $C$ satisfies Arrow's choice axiom, independence of infeasible alternatives, and weak Pareto with a complete $\bar{x}$-restricted feasible set domain. Let $\mathscr{D}_{\bar{x}}$ denote the unique $\bar{x}$-minimal preference domain, i. e. $\mathscr{D}_{\bar{x}}$ is the set of profiles in which everyone agrees that $\bar{x}$ is strictly the worst alternative in $X$. If $\mathscr{D}_{\bar{x}}$ is a strict subset of the actual preference domain $\mathscr{D}, C$ need not be dictatorial, as illustrated by Theorem 1 . However, Theorem 2 implies that $C$ must be dictatorial when restricted to profiles in $\mathscr{D}_{\bar{x}}$. For example, for all $\left\langle R_{i}\right\rangle \in \mathscr{D}_{\bar{x}}, N^{+}\left(x,\left\langle R_{i}\right\rangle\right)=m$ and $N^{-}\left(x,\left\langle R_{i}\right\rangle\right)=0$ for all $x \in X \backslash\{\bar{x}\}$. As a consequence, restricted to $\mathscr{D}_{\bar{x}}$, person one is a dictator for $\tilde{C}^{m}$.

More generally, suppose $X$ contains at least three alternatives not in $Q$ and a social choice function $C$ satisfies Arrow's choice axiom, independence of infeasible alternatives, and weak Pareto with a complete $Q$-restricted feasible set domain. If $\mathscr{D}$ contains two or more $Q$-minimal preference domains, $C$ must be dictatorial when restricted to each $Q$-minimal preference domain, but the dictator can be made conditional on the individual rankings of the alternatives in $Q$.

With an unrestricted preference domain and a complete $Q$-restricted feasible set domain, an immediate consequence of Theorem 2 is that an impossibility theorem is obtained if the axioms in Theorem 1 are strengthened by replacing nondictatorship with anonymity provided that there are at least three alternatives in $X$ not in $Q$. 
Theorem 3. If a social choice function has a complete $Q$-restricted feasible set domain and an unrestricted preference domain and if $\# Q+3 \leq \# X$, then it can not satisfy all of (i) Arrow's choice axiom, (ii) independence of infeasible alternatives, (iii) weak Pareto, and (iv) anonymity.

\section{Independence Conditions on a Restricted Domain}

Hansson's Theorem implies that any social choice function defined on a complete $\bar{x}$-restricted feasible set domain which satisfies Arrow's choice axiom can be rationalized by a social welfare function. In general, requiring the social choice function to also satisfy independence of infeasible alternatives does not imply that such a social welfare function satisfies independence of irrelevant alternatives, which constrains the social ranking of any set of alternatives $A \subset X$ to depend only on the individual rankings of alternatives in $A$. A social welfare function satisfies partial independence of irrelevant alternatives with respect to $\bar{x}$ if the social ranking of any set of alternatives $A \subset X$ depends only on the individual rankings of alternatives in $A \cup\{\bar{x}\} .{ }^{11}$ In general, a social welfare function rationalizing a social choice function need not satisfy this weaker independence condition even if the social choice function satisfies both independence of infeasible alternatives and weak Pareto. For example, if everyone prefers $\bar{x}$ to both $y$ and $z$, the social ranking of $y$ and $z$ is arbitrary. However, with these assumptions, if the preference domain is the $\bar{x}$-minimal preference domain $\mathscr{D}_{\bar{x}}$, the underlying social welfare function does satisfy partial independence of irrelevant alternatives, which on this domain is equivalent to independence of irrelevant alternatives. With weak Pareto, $\bar{x}$ is never chosen, and the social ranking of $y, z \in X$ is uniquely determined from the choice set of $\{\bar{x}, y, z\}$, which is independent of preferences involving infeasible alternatives.

With a finite number of alternatives there is no loss of generality in assuming that the individual preference orderings are representable by utility functions. A social welfare functional maps profiles of utility functions on $X$ into $\mathscr{R}$, the set of orderings of $X{ }^{12}$ In the Arrow problem, individual utilities are ordinally measurable and interpersonally noncomparable. This assumption concerning the measurability and comparability of utilities is formalized by requiring the social welfare functional to be invariant with respect to independent monotone increasing transforms of the utility functions, i.e. if two profiles of utility functions represent the same profile of individual orderings, the functional must map both profiles into the same social ordering. In this framework, the independence conditions can be reformulated without any implicit assumptions concerning the information content of the utility functions. A social welfare functional satisfies independence of irrelevant alternatives if the social rankings of any set of alternatives $A \subset X$ corresponding to two profiles of utility functions are identical whenever each alternative $x \in A$ is assigned the same vector of utility numbers by both profiles. Similarly, partial independence of

\footnotetext{
11 To simplify the exposition, we only consider partial independence with respect to $\bar{x}$ rather than with respect to some set of alternatives $Q$.

12 Surveys of the literature on social welfare functionals may be found in Blackorby, Donaldson, and Weymark [4] and Sen [16]
} 
irrelevant alternatives with respect to $\bar{x}$ is satisfied if the social rankings of any set of alternatives $A \subset X$ are identical whenever each alternative $x \in A \cup\{\bar{x}\}$ is assigned the same vector of utility numbers by both profiles. These conditions are equivalent to their counterparts for social welfare functions if the social welfare functional is invariant to independent monotone increasing transforms of the utility functions.

Requiring a social welfare functional to be invariant with respect to independent positive affine transforms of the utility functions corresponds to the assumption that individual utility functions are cardinally measurable and interpersonally noncomparable. Unlike in the ordinal context, with this information assumption, if all admissible utility profiles represent preferences in $\mathscr{D}_{\bar{x}}$, partial independence of irrelevant alternatives is not equivalent to independence of irrelevant alternatives; for sets of alternatives $A \subset X \backslash\{\bar{x}\}$ the actual utility numbers assigned to $\bar{x}$, not just the utility numbers assigned to elements of $A$, matter in determining the social ordering of $A$.

For cardinally measurable and interpersonally noncomparable utility functions, Roberts [15, Theorem 7] establishes that if the domain of a social welfare functional consists of all profiles of utility functions which represent preference profiles in $\mathscr{D}_{\bar{x}}$ and if the functional satisfies partial independence of irrelevant alternatives and a standard weak Pareto condition, then there must exist a semipositive vector $a$ such that

$$
\prod_{i=1}^{m}\left[U^{i}(x)-U^{i}(\bar{x})\right]^{a_{i}}>\prod_{i=1}^{m}\left[U^{i}(y)-U^{i}(\bar{x})\right]^{a_{i}} \rightarrow x P y
$$

for all $x, y \in X \backslash\{\bar{x}\}$ and for all profiles of utility functions $\left(U^{1}, \ldots, U^{m}\right)$ satisfying the domain restriction, provided $\# X \geq 4 .{ }^{13}$ It is a simple matter to extend Roberts' theorem to the ordinal context. If the social welfare functional must be invariant to ordinal, and not just cardinal, transforms of the utility functions, only one of the $a_{i}$ can be positive; i.e. there must be a dictator. This result is a social welfare functional analogue to Theorem 2 for $Q=\{\bar{x}\}$.

Adding anonymity to Roberts' assumptions implies that the $a_{i}$ in (*) must all be equal, and without loss of generality they can be set equal to one. Thus Roberts has obtained an axiomatization of a social welfare functional which rationalizes the Nash [10] bargaining solution. ${ }^{14}$ The rankings of the alternatives obtained using (*) with each $a_{i}$ equal to one are not invariant to ordinal transforms of the utility functions. This observation can be used to provide an alternative proof of our Theorem 3 with $Q=\{\bar{x}\}$.

The conclusions to be drawn from our analysis are essentially negative. While it is possible to find social choice functions which satisfy all of Arrow's conditions when the preference domain is unrestricted and the feasible set domain is $Q$-restricted, all such rules must exhibit dictatorial features if the $Q$-restricted feasible set domain is complete.

13 Strictly speaking, Roberts works with an unrestricted preference domain and shows that (*) must apply when the functional is restricted to preferences in $\mathscr{D}_{\bar{x}}$.

14 See also Kaneko [8], Kaneko and Nakamura [9], and Osborne [11] for related results. 


\section{References}

1. Arrow KJ (1959) Rational choice functions and orderings. Economica 26:121-127

2. Arrow KJ (1963) Social choice and individual values, second edition. John Wiley and Sons, New York

3. Bailey MJ (1979) The possibility of rational social choice in an economy. J Polit Econ 87: $37-56$

4. Blackorby C, Donaldson D, Weymark JA (1984) Social choice with interpersonal utility comparisons: A diagrammatic introduction. Int Econ Rev 25:327-356

5. Donaldson D, Weymark JA (1985) Social choice in economic environments. Discussion Paper No. 85-20, Department of Economics, University of British Columbia

6. Grether DM, Plott CR (1982) Nonbinary social choice: An impossibility theorem. Rev Econ Stud 49:143-149

7. Hansson B (1968) Choice structures and preference relations. Synthese 18:443-458

8. Kaneko M (1980) An extension of the Nash bargaining problem and the Nash social welfare function. Theory and Decision 12:135-148

9. Kaneko M, Nakamura K (1979) The Nash social welfare function. Econometrica 47:423-435

10. Nash J (1950) The bargaining problem. Econometrica 18:155-162

11. Osborne DK (1976) Irrelevant alternatives and social welfare. Econometrica 44:1001-1015

12. Panda SC (1983) On non-binary social choice. Math Soc Sci 4:73-78

13. Panda SC (1986) Some impossibility results with domain restrictions. J Econ Theory $38: 21-34$

14. Richelson JT (1984) Social choice and the status quo. Public Choice 42:225-234

15. Roberts KWS (1980) Interpersonal comparability and social choice theory. Rev Econ Stud 47: $421-439$

16. Sen AK (1977) On weights and measures: Informational constraints in social welfare analysis. Econometrica 45:1539-1572

17. Suzumura K (1983) Rational choice, collective decisions, and social welfare. Cambridge University Press, Cambridge 